\title{
Efficacy and safety of mitoxantrone hydrochloride liposome injection in Chinese patients with advanced breast cancer: a randomized, open-label, active-controlled, single-center, phase II clinical trial
}

\author{
Leiping Wang ${ }^{1,2}$. Jun $\mathrm{Cao}^{1,2} \cdot$ Chunlei $\mathrm{Li}^{3} \cdot$ Xiaodong Wang ${ }^{3} \cdot$ Yannan $\mathrm{Zhao}^{1,2} \cdot \mathrm{Ting}^{1 \mathrm{Li}^{1,2}} \cdot$ Yiqun $\mathrm{Du}^{1,2}$. \\ Zhonghua Tao $^{1,2} \cdot$ Wenxia Peng $^{1,2} \cdot$ Biyun Wang $^{1,2}$. Jian Zhang ${ }^{1,2}$. Sheng Zhang ${ }^{1,2} \cdot$ Zhonghua Wang $^{1,2}$. \\ Xichun $\mathrm{Hu}^{1,2}$ (1)
}

Received: 27 July 2021 / Accepted: 16 September 2021 / Published online: 11 October 2021

(C) The Author(s) 2021

\begin{abstract}
Summary
Purpose. This trial aimed to evaluate the efficacy and safety of mitoxantrone hydrochloride liposome injection (Lipo-MIT) in advanced breast cancer (ABC). Methods. In this randomized, open-label, active-controlled, single-center, phase II clinical trial, eligible patients were randomized in a ratio of 1:1 to receive Lipo-MIT or mitoxantrone hydrochloride injection (MIT) intravenously. The primary endpoint was objective response rate (ORR). The secondary endpoints were disease control rate (DCR), progression-free survival (PFS), and safety outcomes. Results. Sixty patients were randomized to receive Lipo-MIT or MIT. The ORR was 13.3\% (95\% confidence interval (CI): 3.8-30.7\%) for Lipo-MIT and 6.7\% (95\% CI: 0.8-22.1\%) for MIT. The DCR was 50\% (95\% CI: 31.3-68.7\%) with Lipo-MIT vs. 30\% (95\% CI: 14.7-49.4\%) with MIT. The median PFS was 1.92 months (95\% CI: 1.75-3.61) for Lipo-MIT and 1.85 months (95\% CI: 1.75-2.02) for MIT. The most common toxicity was myelosuppression. Lipo-MIT resulted in an incidence of $86.7 \%$ of leukopenia and $80.0 \%$ of neutropenia, which was marginally superior to MIT (96.7\% and 96.7\%, respectively). Lipo-MIT showed a lower incidence of cardiovascular events (13.3\% vs. $20.0 \%)$ and increased cardiac troponin T (3.3\% vs. $36.7 \%)$; but higher incidence of anemia (76.7\% vs. $46.7 \%)$, skin hyperpigmentation (66.7\% vs. $3.3 \%$ ), and fever (23.3\% vs. $10.0 \%$ ) than MIT. Conclusions The clinical benefit parameters of Lipo-MIT and MIT were comparable. Lipo-MIT provided a different toxicity profile, which might be associated with the altered distribution of the drug. Additional study is needed to elucidate the potential benefit of Lipo-MIT in ABC. Clinical trial registration. This study is registered with ClinicalTrials.gov (No. NCT02596373) on Nov 4, 2015.
\end{abstract}

Keywords Breast neoplasms $\cdot$ Mitoxantrone $\cdot$ Liposomes $\cdot$ Efficacy $\cdot$ Safety

Abbreviations

ABC Advanced breast cancer

AEs Adverse events

Leiping Wang and Jun Cao are co-first authors as they contributed equally to this work. Author order was determined by drawing straws

Xichun $\mathrm{Hu}$

huxichun2017@163.com

1 Department of Medical Oncology, Fudan University Shanghai Cancer Center, Shanghai, China

2 Department of Oncology, Shanghai Medical College, Fudan University, Shanghai, China

3 Department of Medicine, CSPC Zhongqi Pharmaceutical Technology (Shijiazhuang) Co., Ltd, Shanghai, China
CR Complete response

cTnT Cardiac troponin $\mathrm{T}$

DCR Disease control rate

ECOG Eastern Cooperative Oncology Group

FAS Full analysis set

Lipo-MIT Mitoxantrone hydrochloride liposome injection

MIT Mitoxantrone hydrochloride injection

ORR Objective response rate

PD Progressive disease

PFS Progression-free survival

PR Partial response

SD Stable disease

SS Safety set 


\section{Introduction}

Breast cancer has become the most frequently diagnosed cancer worldwide according to the latest global cancer data in 2020 [1]. Among females, breast cancer still tops the list of the most common cancer (approximately 2.3 million new cases accounted for $24.5 \%$ of all cancer cases among women in 2020) and is still the leading cause of cancer death (about six hundred eighty-four thousand deaths in 2020). Despite advances in early diagnosis, 5-10\% of newly diagnosed breast cancer patients were with latestage presentation and metastasis [2]. In addition, 20-30\% of patients with early breast cancer may develop recurrence and metastasis over time [3, 4]. Although metastatic breast cancer remains unlikely to be cured, the meaningful improvement in overall survival was achieved with a median overall survival of three years [5]. Based on molecular profiling, breast cancer could be categorized into three major molecular subtypes: hormone-receptor positive, HER2-positive, and triple-negative breast cancer [6]. In spite of the significant benefit of target therapy and immunotherapy in specific subtypes, chemotherapy is still used as a cornerstone in combination with the other therapies [6, 7]. Chemotherapy is recommended for hormone-insensitive and triple-negative patients with metastatic breast cancer [8]. For HER2-positive patients, the combination of chemotherapy and HER2-targeted agents has shown promising improvement in survival outcomes and was recommended in the ABC 5 guidelines developed by ESO-ESMO [9].

Among many options of chemo-agents, anthracyclines are the most commonly prescribed agents. Mitoxantrone, a synthetic anthracycline anticancer drug, works through inducing DNA lesions, interfering RNA, and inhibiting topoisomerase II to exerting anti-tumor effects [10]. Mitoxantrone-based chemotherapy used to be one of the commonly used chemotherapeutic drugs for breast cancer [11], prostate cancer [12], lymphoma [13], acute leukemia [14], and multiple sclerosis [15] with excellent efficacy. Like many other chemotherapeutic drugs, cardiotoxicity was a major concern for patients treated with mitoxantrone. Several studies have demonstrated the association between mitoxantrone and the increased risk of cardiac dysfunction [16-18]. Due to the black box warnings of cardiotoxicity and a higher risk of developing secondary leukemia, the use of mitoxantrone has been limited, especially in patients who have prior anthracycline therapy $[10,19]$.

The advantages of liposomal formulation stem from its ability to enhance drug stability, sustain release, and target tumor tissues [10, 20]. Therefore, improved antitumor efficacy and reduced toxicity are both expected. The in vivo studies have shown that pegylated liposomal formulation altered the pharmacokinetics and tissue distribution of mitoxantrone, resulting in a more favorable therapeutic response [21, 22]. The previous clinical study demonstrated that much fewer adverse events were observed in the cancer patients in the pegylated mitoxantrone liposome group compared to those using the mitoxantrone at the same dose level, which was a promising improvement in the safety outcomes [23].

The present study was a randomized, open-label, activecontrolled, single-center, phase II clinical trial to evaluate the efficacy and safety of mitoxantrone hydrochloride liposome injection (Lipo-MIT) in Chinese patients with ABC who had previously failed to respond to at least two lines of chemotherapies.

\section{Methods}

\section{Study design}

This was a randomized, open-label, active-controlled, singlecenter, phase II clinical trial conducted in Fudan University Shanghai Cancer Center from Oct 26, 2015, through Apr 6, 2017. The purpose of this study was to assess the efficacy and safety of Lipo-MIT in Chinese patients with ABC. The protocol and the informed consent document were approved by the Ethical Committee of Fudan University Shanghai Cancer Center. The study was conducted in compliance with the principles of Good Clinical Practice and the Declaration of Helsinki. All patients provided the signed informed consent before entering the study. This study was registered in ClinicalTrials.gov: NCT02596373.

The dose selection of Lipo-MIT was based on the results of our phase I study, which demonstrated that Lipo-MIT was safe and effective at 12 to $24 \mathrm{mg} / \mathrm{m}^{2}$ [23]. According to the standard treatment protocol of MIT, patients could receive MIT to a maximum cumulative dose of $160 \mathrm{mg} / \mathrm{m}^{2}$ to limit the risks of cardiotoxicity, and $120 \mathrm{mg} / \mathrm{m}^{2}$ for those who have experienced prior anthracycline therapy [19]. And the conventional treatment consists of 4 to 8 cycles for anthracyclines [24]. To ensure the treatment efficacy, $20 \mathrm{mg} / \mathrm{m}^{2}$ was selected as the recommended dose of Lipo-MIT in our study. This dose selection of Lipo-MIT allows patients to take adequate cycles of chemotherapy, and provides operational flexibility for the dose adjustment (to avoid underdosing). Following label instructions, the use of MIT in this study was at $14 \mathrm{mg} / \mathrm{m}^{2}$ [25].

After a 28-day screening period (Day -28 to Day -1), eligible patients entered a treatment period (Week 1 to Week 32). They were randomized in a ratio of $1: 1$ to receive $20 \mathrm{mg} / \mathrm{m}^{2}$ of Lipo-MIT (CSPC Zhongqi Pharmaceutical Technology (Shijiazhuang) Co., Ltd.) or $14 \mathrm{mg} / \mathrm{m}^{2}$ of mitoxantrone hydrochloride injection (MIT, Sichuan Shenghe 
Pharmaceutical Co., Ltd.) intravenously, once in a 28-day cycle (up to 8 cycles) until disease progression, intolerable toxicity, or death. According to the safety review, a dose adjustment or treatment postponement was permitted at the discretion of the treating physician. Efficacy and safety were recorded appropriately during the whole study.

\section{Study population}

Female patients who aged 18 to 75 years (inclusive), with histo-pathologically and/or cytologically confirmed $A B C$ and failed for at least two lines of chemotherapy regimens previously, had Eastern Cooperative Oncology Group (ECOG) performance status of 0 to 2 , had adequate organ function and bone marrow function, and had at last one measurable lesion with diameter $\geq 10 \mathrm{~mm}$ according to Response Evaluation Criteria in Solid Tumors (RECIST, version 1.1) criteria were recruited in this trial. For patients with hormone receptor-positive breast cancer, an endocrineresistant disease was already developed. Patients with HER2-positive breast cancer were included if they had stopped responding to HER2-targeted therapy or it was not affordable. If patients underwent anthracycline-containing adjuvant chemotherapy before, a more than 12-month interval was required from the last dose of prior anthracycline chemotherapy to the diagnosis of recurrence.

Main exclusion criteria included: patients with a cumulative dose of doxorubicin (or pirarubicin) $>360 \mathrm{mg} / \mathrm{m}^{2}$ or epirubicin $>600 \mathrm{mg} / \mathrm{m}^{2}$, previous mitoxantrone treatment,

Table 1 Demographics and baseline characteristics

\begin{tabular}{|c|c|c|c|}
\hline Characteristic & Lip-MIT (N=30) & $\operatorname{MIT}(\mathbf{N}=30)$ & $P$-value \\
\hline \multicolumn{4}{|l|}{ Sex } \\
\hline Female, N (\%) & $30(100.0)$ & $30(100.0)$ & - \\
\hline Age (years), median (range) & $56.0(27-69)$ & $54.5(44-62)$ & 0.85 \\
\hline \multicolumn{4}{|l|}{ Race or ethnic group, } \\
\hline Chinese/Han, N (\%) & $30(100.0)$ & $30(100.0)$ & - \\
\hline ECOG score, $\mathrm{N}(\%)$ & & & 1.00 \\
\hline 0 & $0(0.0)$ & $1(3.3)$ & \\
\hline 1 & $30(100.0)$ & $29(96.7)$ & \\
\hline Metastatic sites ${ }^{\mathrm{a}}, \mathrm{n}$ & & & 0.91 \\
\hline Visceral & 46 & 43 & \\
\hline Non-visceral & 39 & 37 & \\
\hline Number of metastatic sites, $\mathrm{N}(\%)$ & & & 0.27 \\
\hline 1 & $6(20.0)$ & $6(20.0)$ & \\
\hline 2 & $4(13.3$ & $9(30.0)$ & \\
\hline 3 or more & $20(66.7)$ & $15(50.0)$ & \\
\hline Molecular subtypes, N (\%) & & & 0.51 \\
\hline Hormone-receptor positive & $17(56.7)$ & $23(76.7)$ & \\
\hline HER2-positive & $5(16.7)$ & $3(10.0)$ & \\
\hline Triple negative & $6(20.0)$ & $3(10.0)$ & \\
\hline NA & $2(6.7)$ & $1(3.3)$ & \\
\hline \multicolumn{4}{|l|}{ Previous oncology therapy, N (\%) } \\
\hline Previous surgery & $28(93.3)$ & $29(96.7)$ & 0.99 \\
\hline Previous chemotherapy & $30(100.0)$ & $30(100.0)$ & \\
\hline Anthracycline-containing chemotherapy & $27(90.0)$ & $27(90.0)$ & \\
\hline Previous radiation therapy & $3(10.0)$ & $3(10.0)$ & \\
\hline Previous endocrine therapy & $23(76.7)$ & $25(83.3)$ & \\
\hline Baseline cumulative anthracycline dose $\left(\mathrm{mg} / \mathrm{m}^{2}\right)$, mean $(\mathrm{SD})$ & $295.8(98.74)$ & $288.6(115.98)$ & 0.81 \\
\hline $\begin{array}{l}\text { History of heart diseases, high blood pressure, diabetes mellitus, or high } \\
\text { blood cholesterol, } \mathrm{N}(\%)\end{array}$ & $5(16.7)$ & $11(36.7)$ & 0.26 \\
\hline
\end{tabular}

Data are expressed as counts (percentage) unless otherwise specified

ECOG Eastern Cooperative Oncology Group, Lipo-MIT mitoxantrone hydrochloride liposome injection, MIT mitoxantrone hydrochloride injection, $n$ number of sites, $N$ number of patients, $S D$ standard deviation

${ }^{a}$ A patient may have more than one metastatic site and may have both visceral and non-visceral metastasis 
previous anthracycline treatment for recurrence or metastasis, severe uncontrolled diseases, or other second malignancy.

\section{Efficacy evaluation}

The primary endpoint was objective response rate (ORR), which was defined as the proportion of patients who have a complete response (CR) and partial response (PR). Secondary endpoints were disease control rate (DCR), which was defined as the sum of CR, PR, and stable disease (SD) rates; and progression-free survival (PFS) (the time from randomization to disease progression or death). Efficacy evaluation was carried out according to RECIST (version 1.1) criteria.

Efficacy assessment was conducted with appropriate imageological examinations at baseline and every eight weeks during the treatment period. All CRs and PRs required a repeated confirmatory examination four weeks after the initial assessment. After completion of the treatment, PFS follow-up was conducted every three months until documented disease progression or death (for up to 2 years).

\section{Safety evaluation}

Safety evaluation included adverse events (AEs), changes in vital signs, and clinical laboratory tests (such as blood chemistry test, routine blood test, routine urinalysis, electrocardiogram, and echocardiogram). The severity of AEs was assessed according to the National Cancer Institute Common Terminology Criteria for Adverse Events (NCI-CTCAE, version 4.0).

Safety evaluation was conducted from the signing of the informed consent form to 30 days after completion of the treatment. Cardiotoxicity follow-up for patients with dose adjustment due to cardiovascular AEs was performed by echocardiogram once every three months after completion of the treatment (for up to 2 years).

\section{Statistical analysis}

Patients who successfully received at least one dose of study drug with an adequate baseline assessment were included in the full analysis set (FAS); FAS was the primary analysis set
Fig. 1 A waterfall plot of the best percent change from baseline in the sum of the diameters of the target lesions. Panel a shows the result of Lipo-MIT group, Panel b shows the result of MIT group
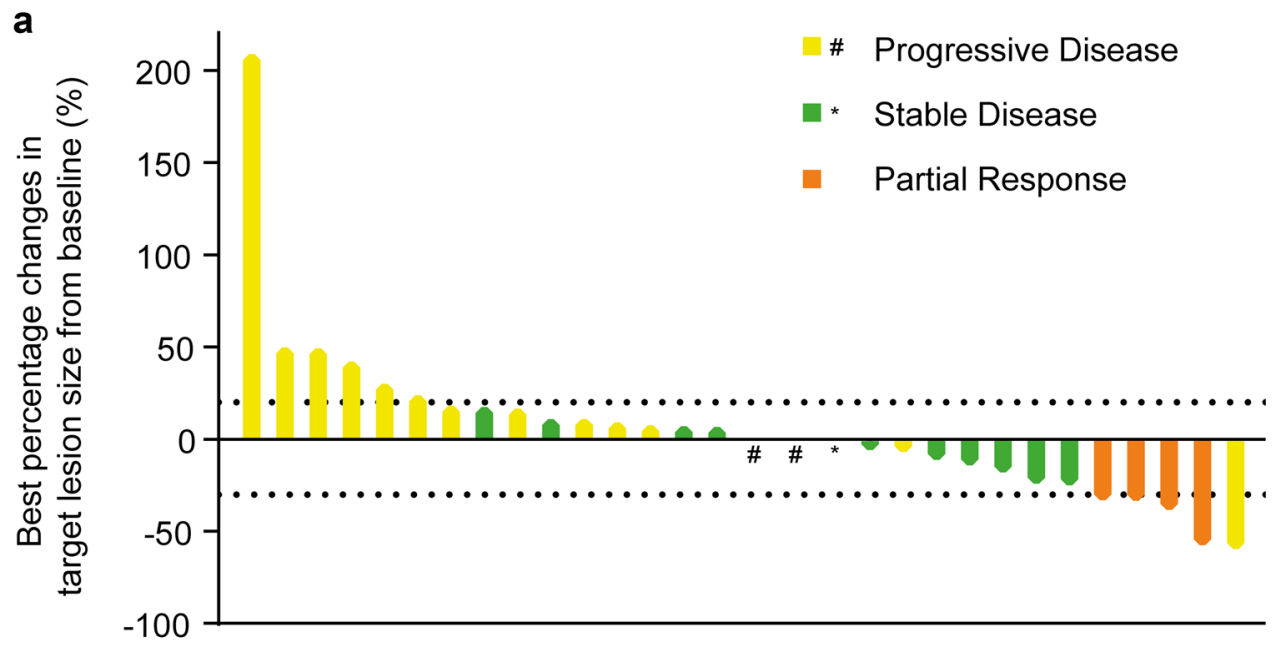

b

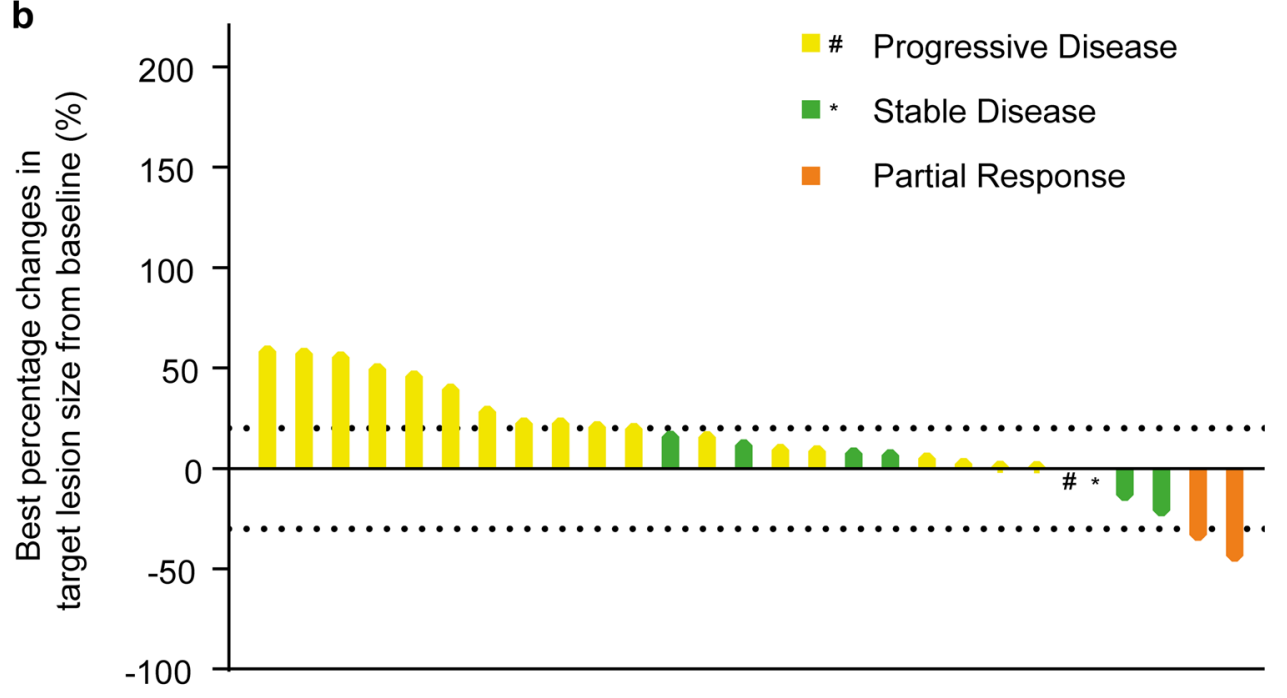


for efficacy. Patients who received at least one dose of study drug were included in the safety set (SS); SS was the primary analysis set for safety.

Because of the limited understanding of the new liposomal treatment in Chinese patients, the initial sample size was set at 60 without statistical estimation. The randomization sequences were generated by an independent statistician using SAS software (version 9.4). The sequential numbering method was used for drug allocation.

All statistical analyses were performed using SAS (version 9.4). All statistical tests were 2-tailed at $\alpha=0.05$ level for significance. Continuous variables were described by mean, standard deviation, or median (minimum and maximum) values as appropriate, and differences were evaluated by $t$-test or $t$ 'test. Categorical or ranked data were described by count and percentage, and differences were evaluated by Chi-square test or Fisher's exact test. ORR and DCR were summarized for each group along with $95 \%$ confidence intervals (CI). Kaplan-Meier analysis was used to estimate the median PFS and the corresponding 95\% CI, log-rank test to compare PFS between the two groups, and Cox proportional-hazards model to estimate the hazard ratio and the corresponding 95\% CI. Subgroup analysis was conducted according to the molecular subtypes and liver metastases. Adverse events used the Medical Dictionary for Regulatory Activities for coding (MedDRA, version 21.0).

\section{Results}

\section{Study population and drug administration}

Between October 2015 and April 2017, a total of 60 patients were randomized in a ratio of $1: 1$ to receive $20 \mathrm{mg} / \mathrm{m}^{2}$ of Lipo-MIT or $14 \mathrm{mg} / \mathrm{m}^{2}$ of MIT intravenously. The CONSORT diagram of the patient's deposition is shown in Supplementary Fig. 1. The demographics and baseline characteristics of the patients are summarized in Table 1. The important characteristics of patients were well balanced between the two groups. The median age was 56 (range 44-62). The majority of patients (67\%) had a hormone-receptor-positive disease, $15 \%$ of patients had a triple-negative disease, and $13 \%$ had a HER2-positive disease. And all patients had been heavily pretreated; 27 patients in each group had received prior anthracyclines (epirubicin, or doxorubicin) therapy. The baseline average cumulative dose of anthracyclines was $295.8 \mathrm{mg} / \mathrm{m}^{2}$ and $288.6 \mathrm{mg} / \mathrm{m}^{2}$, without statistical significance between groups $(P>0.05)$ (Table 1).

The drug administration information is summarized in Supplementary Table 1 . The median number of cycles delivered was two (range 1-6) in the Lipo-MIT group and two (range 1-8) in the MIT group. The percentage of patients who completed four treatment cycles was higher in the Lipo-MIT group (36.7\% in the Lipo-MIT group vs. $23.3 \%$ in the MIT group).

\section{Efficacy}

All patients in this study were evaluated for response. The best percentage changes from baseline and the best overall response are shown in Fig. 1. Overall, the ORR was $13.3 \%$ (95\% CI: $3.8-30.7 \%$ ) and DCR was $50 \%$ (95\% CI: $31.3-68.7 \%)$ in the Lipo-MIT group, with 4 (13.3\%) patients achieved PR and 11 (36.7\%) SD (Table 2). The ORR in the MIT group was 6.7\% (95\% CI: $0.8-22.1 \%)$ and the DCR was 30\% (95\% CI: $14.7-49.4 \%$ ) (Table 2). The ORR favored the Lipo-MIT group, but the difference was not statistically significant $(P>0.05)$. Figure 2 shows a swimmer plot of the time to best responses and the treatment duration. The median PFS was 1.92 (95\% CI: 1.75-3.61) months in the Lipo-MIT group versus 1.85 (95\% CI: 1.75-2.02) months in the MIT group (Supplementary Fig. 2). The hazard ratio of PFS was 1.13 (95\% CI: 0.64-1.99) for the Lipo-MIT group versus the MIT group. No significant difference was found (Log-rank P > 0.05, Supplementary Fig. 2). In subgroup analysis based on the molecular subtypes and liver metastases, we found that the clinical benefit parameters were consistent with the overall result across different subgroups; no statistically significant differences were seen between groups in the response rate and the median PFS (Supplementary Table 2).

\section{Safety}

The summary of AEs is listed in Table 3. Totally, 1751 AEs in 15 system organ classes (SOCs) were reported in

Table 2 Tumor response

\begin{tabular}{llll}
\hline Efficacy measurement & Lipo-MIT & MIT & $P$-value \\
\hline Best overall response (\%) & & & 0.14 \\
CR & $0.0(0 / 30)$ & $0.0(0 / 30)$ & \\
PR & $13.3(4 / 30)$ & $6.7(2 / 30)$ & \\
SD & $36.7(11 / 30)$ & $23.3(7 / 30)$ & \\
PD & $50.0(15 / 30)$ & $63.3(19 / 30)$ & \\
NE & $0.0(0 / 30)$ & $6.7(2 / 30)$ & \\
ORR (\%) & $13.3(4 / 30)$, & $6.7(2 / 30)$, & 0.67 \\
& CI: $3.8-30.7$ & CI: $0.8-22.1$ & \\
DCR $(\%)$ & $50.0(15 / 30)$, & $30.0(9 / 30)$, & 0.11 \\
& CI: $31.3-68.7$ & CI: $14.7-49.4$ & \\
\hline
\end{tabular}

Data are expressed as percentage (counts) unless otherwise specified. DCR: defined as the percentage of patients with $\mathrm{CR}$ or PR or $\mathrm{SD}$. DCR is described by percentage (number of CRs + PRs + SDs / number of patients) and its CI. ORR: defined as the percentage of patients with CR or PR. ORR is described by percentage (number of $\mathrm{CRs}+\mathrm{PRs}$ / number of patients) and its CI

$C I$ 95\% confidence interval, $C R$ complete response, DCR disease control rate, Lipo-MIT mitoxantrone hydrochloride liposome injection, $M I T$ mitoxantrone hydrochloride injection, $N E$ Not evaluable, $O R R$ overall response rate, $P D$ progress disease, $P R$ partial response, $S D$ stable disease 
Fig. 2 A swimmer plot of the objective responses according to RECIST (version 1.1) from the start of treatment to the end of treatment. Panel a shows the result of Lipo-MIT group, Panel b shows the result of MIT group. Each horizontal bar represents one patient. The treatment duration was defined as the time from the first treatment to the time of documented progression, withdrawal, death, or completion of full course of treatment. PD: progressive disease; PR: partial response; SD: stable disease; NA: not available a

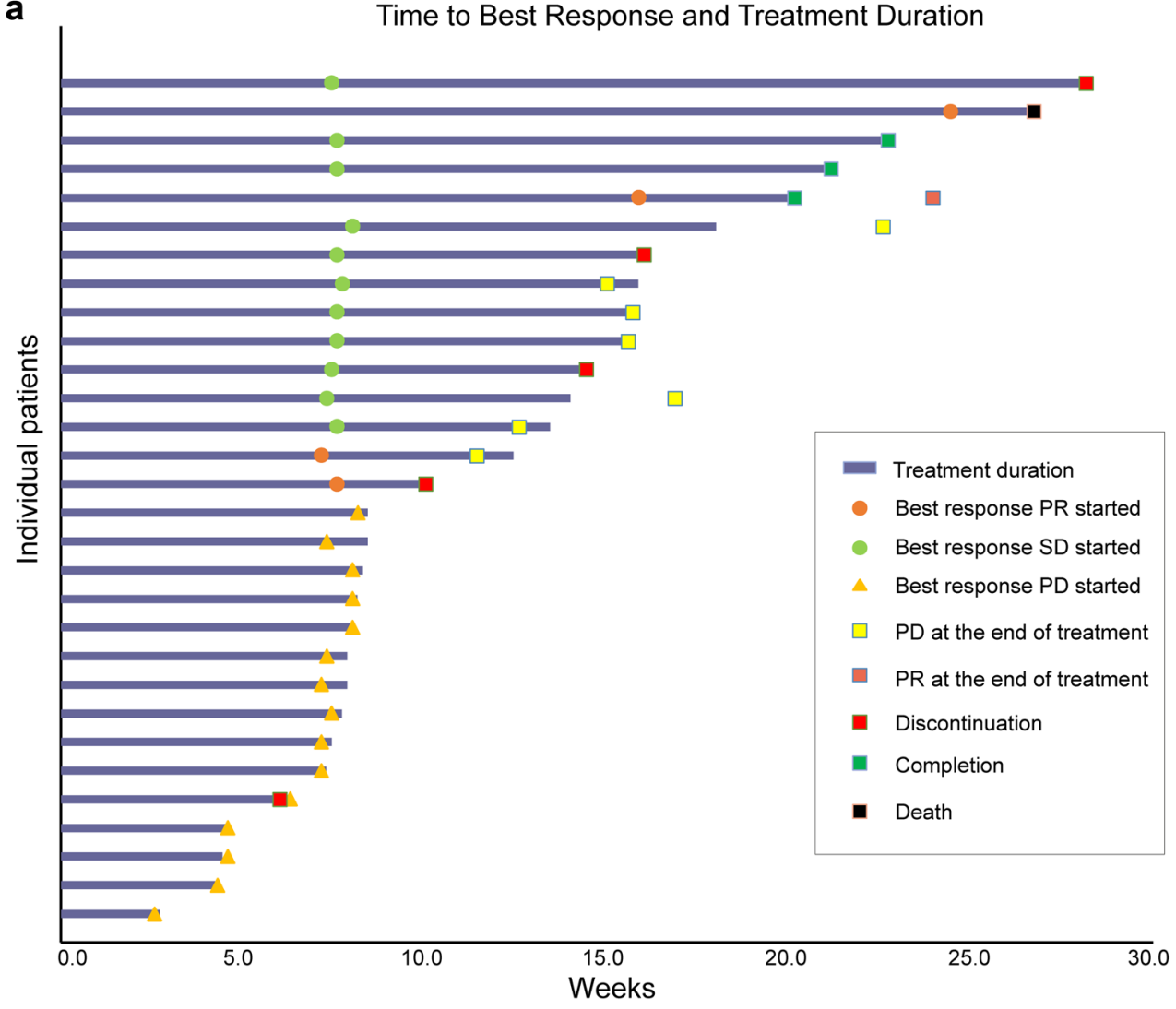

b

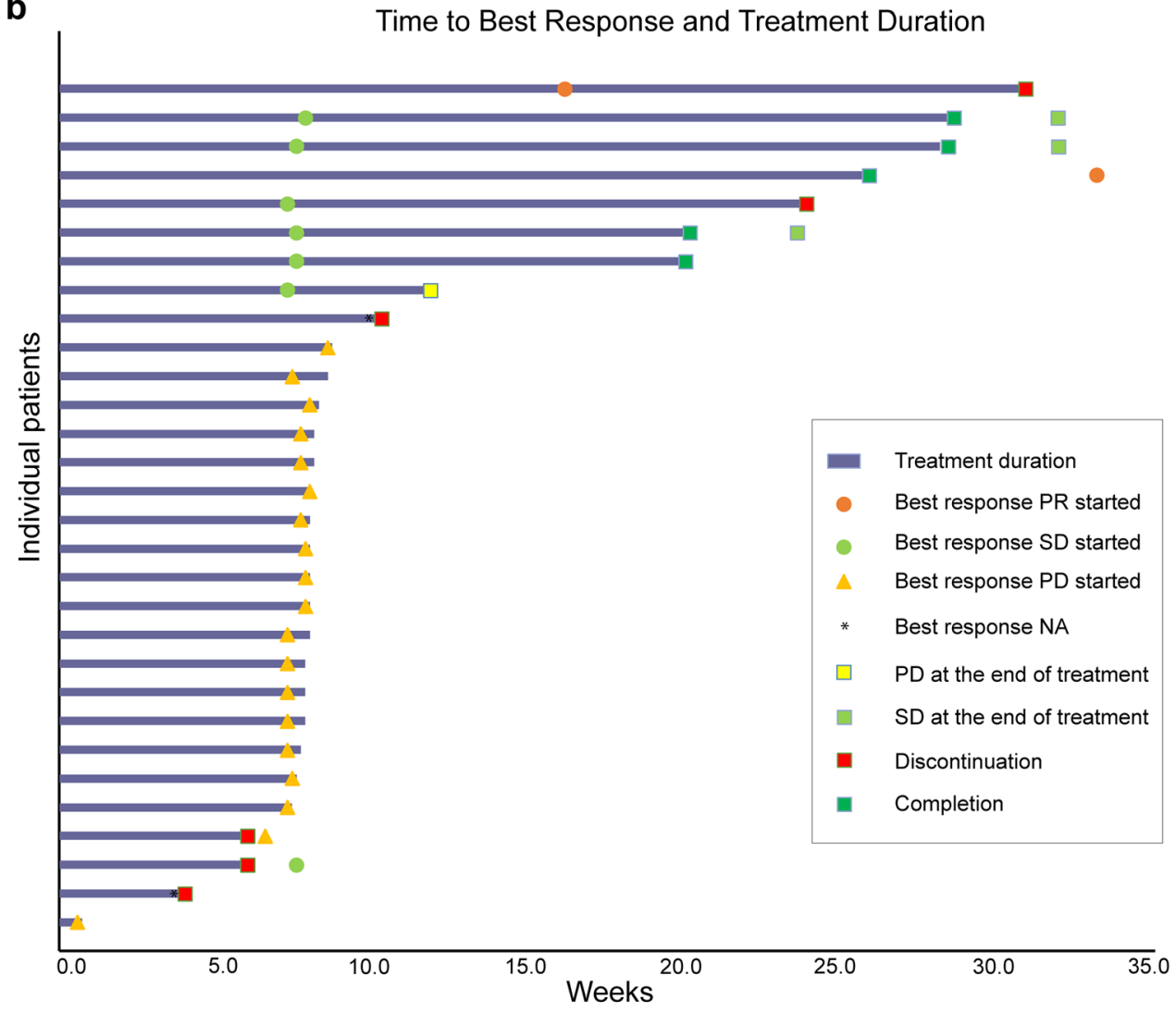


Table 3 Summary of Adverse Events (AEs)

\begin{tabular}{|c|c|c|c|c|c|c|}
\hline \multirow[t]{2}{*}{ AEs ${ }^{a}$} & \multicolumn{3}{|c|}{$\begin{array}{l}\text { Lipo-MIT }(\mathbf{N}=30) \\
\text { N (\%) }\end{array}$} & \multicolumn{3}{|c|}{$\begin{array}{l}\text { MIT }(N=30) \\
N(\%)\end{array}$} \\
\hline & All Grades & Grade 3 & Grade 4 & All Grades & Grade 3 & Grade 4 \\
\hline Any AEs & $30(100.0)$ & $23(76.7)$ & $7(23.3)$ & $30(100.0)$ & $29(96.7)$ & $8(26.7)$ \\
\hline \multicolumn{7}{|l|}{ Hematological AEs } \\
\hline Leukopenia & $26(86.7)$ & $15(50.0)$ & $2(6.7)$ & $29(96.7)$ & $16(53.3)$ & $3(10.0)$ \\
\hline Neutropenia & $24(80.0)$ & $15(50.0)$ & $3(10.0)$ & $29(96.7)$ & $20(66.7)$ & $8(26.7)$ \\
\hline Anemia & $23(76.7)$ & $6(20.0)$ & $1(3.3)$ & $14(46.7)$ & $2(6.7)$ & $0(0.0)$ \\
\hline Thrombocytopenia & $17(56.7)$ & $4(13.3)$ & $5(16.7)$ & $16(53.3)$ & $2(6.7)$ & $0(0.0)$ \\
\hline \multicolumn{7}{|l|}{ Non-hematological AEs } \\
\hline Skin hyperpigmentation & $20(66.7)$ & $0(0.0)$ & $0(0.0)$ & $1(3.3)$ & $0(0.0)$ & $0(0.0)$ \\
\hline Increased conjugated bilirubin & $16(53.3)$ & $0(0.0)$ & $2(6.7)$ & $17(56.7)$ & $2(6.7)$ & $0(0.0)$ \\
\hline Increased AST & $12(40.0)$ & $1(3.3)$ & $0(0.0)$ & $16(53.3)$ & $2(6.7)$ & $0(0.0)$ \\
\hline Increased BNP & $10(33.3)$ & $0(0.0)$ & $0(0.0)$ & $10(33.3)$ & $0(0.0)$ & $0(0.0)$ \\
\hline Increased total bilirubin & $9(30.0)$ & $1(3.3)$ & $1(3.3)$ & $6(20.0)$ & $0(0.0)$ & $0(0.0)$ \\
\hline Increased ALT & $6(20.0)$ & $0(0.0)$ & $0(0.0)$ & $8(26.7)$ & $2(6.7)$ & $0(0.0)$ \\
\hline Increased cTnT & $1(3.3)$ & $0(0.0)$ & $0(0.0)$ & $11(36.7)$ & $0(0.0)$ & $0(0.0)$ \\
\hline Fever & $7(23.3)$ & $1(3.3)$ & $0(0.0)$ & $3(10.0)$ & $0(0.0)$ & $0(0.0)$ \\
\hline Fatigue & $6(20.0)$ & $1(3.3)$ & $0(0.0)$ & $4(13.3)$ & $1(3.3)$ & $0(0.0)$ \\
\hline Hypokalemia & $3(10.0)$ & $1(3.3)$ & $1(3.3)$ & $3(10.0)$ & $0(0.0)$ & $1(3.3)$ \\
\hline Increased GGT & $3(10.0)$ & $2(6.7)$ & $0(0.0)$ & $3(10.0)$ & $1(3.3)$ & $0(0.0)$ \\
\hline Hypertension & $2(6.7)$ & $1(3.3)$ & $0(0.0)$ & $2(6.7)$ & $1(3.3)$ & $0(0.0)$ \\
\hline Pneumonitis & $2(6.7)$ & $1(3.3)$ & $0(0.0)$ & $2(6.7)$ & $1(3.3)$ & $0(0.0)$ \\
\hline Hypocalcemia & $2(6.7)$ & $1(3.3)$ & $0(0.0)$ & $0(0.0)$ & $0(0.0)$ & $0(0.0)$ \\
\hline Hyponatremia & $2(6.7)$ & $1(3.3)$ & $0(0.0)$ & $0(0.0)$ & $0(0.0)$ & $0(0.0)$ \\
\hline Soft tissue infection & $2(6.7)$ & $1(3.3)$ & $0(0.0)$ & $0(0.0)$ & $0(0.0)$ & $0(0.0)$ \\
\hline Hypophosphatemia & $1(3.3)$ & $0(0.0)$ & $1(3.3)$ & $0(0.0)$ & $0(0.0)$ & $0(0.0)$ \\
\hline Decreased serum phosphorus & $1(3.3)$ & $1(3.3)$ & $0(0.0)$ & $0(0.0)$ & $0(0.0)$ & $0(0.0)$ \\
\hline Urethral infection & $1(3.3)$ & $1(3.3)$ & $0(0.0)$ & $0(0.0)$ & $0(0.0)$ & $0(0.0)$ \\
\hline Chronic bronchitis & $0(0.0)$ & $0(0.0)$ & $0(0.0)$ & $1(3.3)$ & $1(3.3)$ & $0(0.0)$ \\
\hline Febrile neutropenia & $0(0.0)$ & $0(0.0)$ & $0(0.0)$ & $1(3.3)$ & $1(3.3)$ & $0(0.0)$ \\
\hline
\end{tabular}

Data are expressed as counts (percentage) unless otherwise specified

$A L T$ alanine aminotransferase, $A S T$ aspartate aminotransferase, $B N P$ Brain natriuretic peptide, $c T n T$ cardiac troponin T, GGT Gamma glutamyl transferase, Lipo-MIT mitoxantrone hydrochloride liposome injection, MIT mitoxantrone hydrochloride injection, $N$ number of patients

ancluded AEs are adverse events of any grade that occurred in at least $20 \%$ of the patients or grade $3-4$ adverse events

60 enrolled patients, with the most frequent being blood and lymphatic system disorders. All patients in the LipoMIT group and the MIT group experienced at least one AE. Grade 3-4 AEs occurred.

more frequently in the MIT group compared to the LipoMIT group (grade 3-4 AEs reported by 29 patients with MIT, 23 with Lipo-MIT). Eight (26.7\%) patients in the LipoMIT group discontinued due to an adverse event, while one MIT-treated patient (3.3\%) discontinued due to an adverse event. Nine cases of drug-related serious adverse events (SAEs) occurred in six patients in the Lipo-MIT group; four cases of drug-related SAEs occurred in two patients in the MIT group (Supplementary Table 3). One case of death (due to interstitial pneumonia) was reported in the Lipo-MIT group; the causality was assessed as possibly related to the study drug. The details of SAEs are tabulated in Supplementary Table 3.

Overall, myelosuppression was commonly seen in both groups. The lower incidence of leukopenia and neutropenia (all-grade and grade 3-4) was found in the Lipo-MIT group, and the incidence of anemia and thrombocytopenia (all-grade and grade 3-4) was higher in the Lipo-MIT group. Nonhematological AEs were generally mild to moderate compared to hematological AEs. Skin hyperpigmentation was the most common non-hematologic $\mathrm{AE}$ in patients treated with Lipo-MIT (grade 1-2: 66.7\%) compared to patients with MIT 
(grade 1-2: 3.3\%). Generalized symptoms (fatigue and fever) and the changes of the investigational tests (such as increased conjugated bilirubin, increased aspartate aminotransferase) were reported frequently but generally low-grade in both groups. No increase in the incidence of abnormal liver function was observed in Lipo-MIT group (Table 3).

Cardiovascular AEs were the AEs of special interest in our study. The AEs of cardiac disorders, including ventricular extrasystole, supraventricular extrasystoles, palpitation, sinus tachycardia, and left bundle branch block, were reported by four (13.3\%) patients in the Lipo-MIT group, which was lower than that in the MIT group (seven patients, 23.3\%). All cardiovascular AEs were grade 1. The elevated cardiac troponin T (cTnT) level, a cardiotoxicity biomarker, was detected in one patient (3.3\%) in the Lipo-MIT group, which was much lower than that in the MIT group (11 patients, 36.7\%) (Table 3).

\section{Discussion}

Despite the fast advances in treatment options for breast cancer patients, anthracycline-based treatment is still an active and preferable option in clinical practice. The conventional mitoxantrone is effective in the treatment of $A B C$ [26]. In the Chinese Expert Consensus on Anthracyclines Treatment of Breast Cancer [25], the use of anthracyclines alone or in combination is recommended in patients with $\mathrm{ABC}$ who are not resistant to anthracycline or have not exceeded the cumulative dose limit. James Neidhart and colleagues reported that the efficacy of mitoxantrone was comparable with doxorubicin in breast cancer patients, with more favorable safety outcomes [27]. However, the usefulness of mitoxantrone was hampered by its cardiotoxicity and myelosuppression.

It is logical to use a liposomal formulation to improve the safety profiles and enhance the anti-tumor effects via permeability and retention effect [28]. A phase II clinical trial of liposomal mitoxantrone was previously conducted in German ABC patients but failed (only $1 / 17$ patients had a PR) [29]. The explanation of this failure from the authors was the drug leaked from the liposomal bilayer and inadequate circulation time [29]. The modifications of the lipid bilayer of the liposome are undoubtedly crucial for the effectiveness of liposomal formulations [30]. Our Lipo-MIT was prepared by the pegylated hydrogenated soy phosphatidylcholine/cholesterol (HSPC/chol) with a good encapsulation efficiency, which was stable over $60 \mathrm{~min}$ at $60{ }^{\circ} \mathrm{C}$ and over six months at $2-8{ }^{\circ} \mathrm{C}$. The improved circulation time was observed in both the animal model and phase I study [23, 31, 32]. The particle size of Lipo-MIT was down to $60 \mathrm{~nm}$, resulting in a preferential accumulation into tumor tissue in in vivo studies and a fast drug-release rate in in vitro studies [33].
In general, the patients who experienced failure of the first two lines of chemotherapies may have a worse prognosis in the subsequent treatment [34]. In a large retrospective study conducted by Porkka et al., the pooled ORR for the second-line and higher-line treatment was $11 \%$ for $\mathrm{ABC}$ patients [35]. The ORR ( $>2$ lines) in our study was $13.3 \%$ in the Lipo-MIT group, which was comparable with the previous report. However, we did not observe a significant difference in ORR between the Lipo-MIT and MIT groups (13.3\% vs. $6.7 \%, \mathrm{P}>0.05$ ). Similar results have been seen in other studies on liposomal anthracyclines. A phase III trial showed comparable efficacy of liposomal doxorubicin versus doxorubicin in metastatic breast cancer treatment (ORR were $33 \%$ with liposomal doxorubicin and 38\% with doxorubicin) [36]. A lower ORR was observed in our study, which was probably due to our inclusion of heavily pretreated patients who failed the prior anthracyclines treatment.

It was worth noticing that the incidence of skin hyperpigmentation was much higher in the Lipo-MIT group than in the MIT group. And the one patient with skin hyperpigmentation in MIT group was assessed as not related to the study drug. In contrast, all the cases of skin hyperpigmentation in the Lipo-MIT group were related to the study drug. However, no patient needed to reduce the dosage, discontinued the medication, or withdrew from the trial due to skin hyperpigmentation. Medical attention and treatments were not needed to resolve this condition. We speculated that the occurrence of skin hyperpigmentation might be related to the characteristics of drug formulation or tissue distribution.

In the previous phase III of liposomal doxorubicin, the risk of cardiotoxicity with liposomal doxorubicin was much lower than that with conventional doxorubicin in patients with metastatic breast cancer [36]. Our safety results were consistent with the previous study by showing a smaller proportion of patients with cardiovascular AEs. And the incidence of increased cTnT was much lower in the LipoMIT group than in the MIT group (3.3\% vs. $36.7 \%)$. The mechanism of reduced cardiotoxicity of liposomal mitoxantrone might be due to the lower peak concentrations [23], larger area under the concentration-time curve [21-23, 33], prolonged half-life [22], and reduced heart tissue distribution [22]. Myelosuppression was frequently seen in our study. Overall, the incidence of hematological AEs was similar between groups. But more hematological SAEs were reported in the Lipo-MIT group (seven cases with Lipo-MIT, one case with MIT). The comparative safety of Lipo-MIT and MIT was still inconclusive.

One major limitation of our study was the small sample size. Because this was the first study to evaluate the efficacy and safety of Lipo-MIT in Chinese patients with $\mathrm{ABC}$, the sample size was determined without any statistical estimation. Several improvements with LipoMIT in efficacy endpoints and safety outcomes were 
not statistically significant due to the effects of small sample size.

In conclusion, this study provided additional information about the Lipo-MIT in the ABC patients, which indicated a potential advantage in efficacy and cardiovascular safety. But a statistically significant difference of LipoMIT over MIT in ABC was not established in this study. It is worthwhile to further elucidate the risk and benefit of Lipo-MIT as an alternative to MIT in ABC setting.

Supplementary information The online version contains supplementary material available at https://doi.org/10.1007/s10637-021-01182-7.

Acknowledgements We would like to thank all patients/families who participated in this study, investigators, and support staff. This work was supported by the National Key R\&D Program of China (2017YFA0205604) and CSPC Zhongqi Pharmaceutical Technology (Shijiazhuang) Co., Ltd.. We also would like to express our appreciation to Jing Yuan, Sheng Hao, Zhibin Meng, and Kun Lou for assistance with the data management and statistical analysis. The editorial and writing assistance was provided by Cuixia Gao, Chang Liu, Lei Wang, and Zijian Hu.

Author's contributions Leiping Wang, Jun Cao and Xichun Hu contributed to the conception and design of the study. Leiping Wang, Jun Cao, and Xichun $\mathrm{Hu}$ were involved in the acquisition, analysis, and interpretation of the data. Leiping Wang, Xichun Hu, Yannan Zhao, Ting Li, Jun Cao, Yiqun Du, Zhonghua Tao, Wenxia Peng, Biyun Wang, Jian Zhang, Zhonghua Wang, Sheng Zhang and Xiaodong Wang provided administrative, technical, and material support. Leiping Wang, Xichun $\mathrm{Hu}$, Xiaodong Wang and Chunlei Li provided study supervision. All authors participated in the discussion and approved the manuscript as submitted.

Funding The research was supported by the National Key R\&D Program of China (2017YFA0205604). The trial was funded by CSPC Zhongqi Pharmaceutical Technology (Shijiazhuang) Co., Ltd.

Data availability The datasets generated during and/or analyzed during the current study are available from the corresponding author on reasonable request.

Code availability All statistical analyses were performed using SAS software (version 9.4).

\section{Declarations}

Ethical approval All procedures performed in studies involving human participants were in accordance with the ethical standards of the institutional research committee and with the 1964 Helsinki declaration and its later amendments or comparable ethical standards.

Consent to participate Informed consent was obtained from all individual participants included in the study.

Consent for publication Participants signed informed consent regarding publishing their data.

Conflicts of interest Chunlei $\mathrm{Li}$, and Xiaodong Wang are staff of CSPC ZhongQi Pharmaceutical Technology (Shijiazhuang) Co., Ltd. The others declare that they have no conflicts of interest.
Open Access This article is licensed under a Creative Commons Attribution 4.0 International License, which permits use, sharing, adaptation, distribution and reproduction in any medium or format, as long as you give appropriate credit to the original author(s) and the source, provide a link to the Creative Commons licence, and indicate if changes were made. The images or other third party material in this article are included in the article's Creative Commons licence, unless indicated otherwise in a credit line to the material. If material is not included in the article's Creative Commons licence and your intended use is not permitted by statutory regulation or exceeds the permitted use, you will need to obtain permission directly from the copyright holder. To view a copy of this licence, visit http://creativecommons.org/licenses/by/4.0/.

\section{References}

1. Sung H, Ferlay J, Siegel RL, Laversanne M, Soerjomataram I, Jemal A, Bray F (2021) Global cancer statistics 2020: GLOBOCAN estimates of incidence and mortality worldwide for 36 cancers in 185 countries. CA Cancer J Clin. https://doi.org/10.3322/ caac. 21660

2. Cardoso F, Harbeck N, Fallowfield L, Kyriakides S, Senkus E, Group EGW (2012) Locally recurrent or metastatic breast cancer: ESMO Clinical Practice Guidelines for diagnosis, treatment and follow-up. Ann Oncol 23 Suppl 7:vii11-19. https://doi.org/ 10.1093/annonc/mds232

3. Claessens AKM, Ibragimova KIE, Geurts SME, Bos M, Erdkamp FLG, Tjan-Heijnen VCG (2020) The role of chemotherapy in treatment of advanced breast cancer: an overview for clinical practice. Crit Rev Oncol Hematol 153:102988. https://doi.org/10.1016/j. critrevonc.2020.102988

4. Liang Y, Zhang H, Song X, Yang Q (2020) Metastatic heterogeneity of breast cancer: Molecular mechanism and potential therapeutic targets. Semin Cancer Biol 60:14-27. https://doi.org/10.1016/j. semcancer.2019.08.012

5. Caswell-Jin JL, Plevritis SK, Tian L, Cadham CJ, Xu C, Stout NK, Sledge GW, Mandelblatt JS, Kurian AW (2018) Change in Survival in Metastatic Breast Cancer with Treatment Advances: Meta-Analysis and Systematic Review. JNCI Cancer Spectr 2 (4):pky062. https://doi.org/10.1093/jncics/pky062

6. Waks AG, Winer EP (2019) Breast Cancer Treatment: A Review. JAMA 321(3):288-300. https://doi.org/10.1001/jama.2018.19323

7. García-Aranda M, Redondo M (2019) Immunotherapy: A Challenge of Breast Cancer Treatment. Cancers (Basel) 11(12):1822. https://doi.org/10.3390/cancers11121822

8. Chacon Lopez-Muniz JI, de la Cruz ML, Gavila Gregori J, Martinez Duenas E, Oliveira M, Segui Palmer MA, Alvarez Lopez I, Antolin Novoa S, Bellet Ezquerra M, Lopez-Tarruella Cobo S (2019) SEOM clinical guidelines in advanced and recurrent breast cancer (2018). Clin Transl Oncol 21(1):31-45. https://doi.org/10.1007/ s12094-018-02010-w

9. Cardoso F, Paluch-Shimon S, Senkus E, Curigliano G, Aapro MS, Andre F, Barrios CH, Bergh J, Bhattacharyya GS, Biganzoli L, Boyle F, Cardoso MJ, Carey LA, Cortes J, El Saghir NS, Elzayat M, Eniu A, Fallowfield L, Francis PA, Gelmon K, Gligorov J, Haidinger R, Harbeck N, Hu X, Kaufman B, Kaur R, Kiely BE, Kim SB, Lin NU, Mertz SA, Neciosup S, Offersen BV, Ohno S, Pagani O, Prat A, Penault-Llorca F, Rugo HS, Sledge GW, Thomssen C, Vorobiof DA, Wiseman T, Xu B, Norton L, Costa A, Winer EP (2020) 5th ESO-ESMO international consensus guidelines for advanced breast cancer (ABC 5). Ann Oncol 31(12):1623-1649. https://doi.org/10. 1016/j.annonc.2020.09.010

10. Evison BJ, Sleebs BE, Watson KG, Phillips DR, Cutts SM (2016) Mitoxantrone, More than Just Another Topoisomerase II Poison. Med Res Rev 36(2):248-299. https://doi.org/10.1002/med.21364 
11. Hainsworth JD, Andrews MB, Johnson DH, Greco FA (1991) Mitoxantrone, fluorouracil, and high-dose leucovorin: an effective, well-tolerated regimen for metastatic breast cancer. J Clin Oncol 9(10):1731-1735. https://doi.org/10.1200/JCO.1991.9.10.1731

12. Basch EM, Scholz M, de Bono JS, Vogelzang N, de Souza P, Marx G, Vaishampayan U, George S, Schwarz JK, Antonarakis ES, O'Sullivan JM, Kalebasty AR, Chi KN, Dreicer R, Hutson TE, Dueck AC, Bennett AV, Dayan E, Mangeshkar M, Holland J, Weitzman AL, Scher HI (2019) Cabozantinib Versus Mitoxantrone-prednisone in Symptomatic Metastatic Castrationresistant Prostate Cancer: A Randomized Phase 3 Trial with a Primary Pain Endpoint. Eur Urol 75(6):929-937. https://doi.org/ 10.1016/j.eururo.2018.11.033

13. Nastoupil LJ, McLaughlin P, Feng L, Neelapu SS, Samaniego F, Hagemeister FB, Ayala A, Romaguera JE, Goy AH, Neal E, Wang M, Fayad L, Fanale MA, Oki Y, Westin JR, Rodriguez MA, Cabanillas F, Fowler NH (2017) High ten-year remission rates following rituximab, fludarabine, mitoxantrone and dexamethasone (R-FND) with interferon maintenance in indolent lymphoma: Results of a randomized Study. Br J Haematol 177(2):263-270. https://doi.org/10.1111/bjh.14541

14. Advani AS, Cooper B, Visconte V, Elson P, Chan R, Carew J, Wei W, Mukherjee S, Gerds A, Carraway H, Nazha A, Hamilton B, Sobecks R, Caimi P, Tomlinson B, Malek E, Little J, Miron A, Pink J, Maciejewski J, Unger A, Kalaycio M, de Lima M, Sekeres MA (2019) A Phase I/II Trial of MEC (Mitoxantrone, Etoposide, Cytarabine) in Combination with Ixazomib for Relapsed Refractory Acute Myeloid Leukemia. Clin Cancer Res 25(14):42314237. https://doi.org/10.1158/1078-0432.CCR-18-3886

15. Martinelli Boneschi F, Vacchi L, Rovaris M, Capra R, Comi G (2013) Mitoxantrone for multiple sclerosis. Cochrane Database Syst Rev (5). https://doi.org/10.1002/14651858.CD002127.pub3

16. Feijen EAM, Leisenring WM, Stratton KL, Ness KK, van der Pal HJH, van Dalen EC, Armstrong GT, Aune GJ, Green DM, Hudson MM, Loonen J, Oeffinger KC, Robison LL, Yasui Y, Kremer LCM, Chow EJ (2019) Derivation of Anthracycline and Anthraquinone Equivalence Ratios to Doxorubicin for Late-Onset Cardiotoxicity. JAMA Oncol 5(6):864-871. https://doi.org/10. 1001/jamaoncol.2018.6634

17. Shaikh AY, Suryadevara S, Tripathi A, Ahmed M, Kane JL, Escobar J, Cerny J, Nath R, McManus DD, Shih J, McGuiness ME, Tighe DA, Meyer TE, Ramanathan M, Aurigemma GP (2016) Mitoxantrone-Induced Cardiotoxicity in Acute Myeloid Leukemia-A Velocity Vector Imaging Analysis. Echocardiography 33(8):11661177. https://doi.org/10.1111/echo.13245

18. Smith LA, Cornelius VR, Plummer CJ, Levitt G, Verrill M, Canney $P$, Jones A (2010) Cardiotoxicity of anthracycline agents for the treatment of cancer: systematic review and meta-analysis of randomised controlled trials. BMC Cancer 10:337. https://doi.org/10.1186/ 1471-2407-10-337

19. Chinese society of clinical oncology, Chinese society of hematology (2013) Guidelines for the prevention and treatment of cardiotoxicity with anthracyclines (Edition 2013). Chinese Clinical Oncol 18 (10):925-934. (in Chinese). https://doi.org/10.3969/j. issn.1009-0460.2013.10.014

20. Paola M, Franco D, Luigi C (2012) PEGylation of Proteins and Liposomes: a Powerful and Flexible Strategy to Improve the Drug Delivery. Curr Drug Metab 13(1):105-119. https://doi.org/10. 2174/138920012798356934

21. Li C, Zhao X, Deng C, Wang C, Wei N, Cui J (2014) Pegylated liposomal mitoxantrone is more therapeutically active than mitoxantrone in L1210 ascitic tumor and exhibits dose-dependent activity saturation effect. Int J Pharm 460(1):165-172. https://doi.org/ 10.1016/j.ijpharm.2013.10.023

22. Wang CX, Li CL, Zhao X, Yang HY, Zhang L (2010) Pharmacodynamics, pharmacokinetics and tissue distribution of liposomal mitoxantrone hydrochloride. Acta Pharmaceutica Sinica 45(12):1565-1569 ((in Chinese))

23. Yang J, Shi Y, Li C, Gui L, Zhao X, Liu P, Han X, Song Y, Li N, Du P, Zhang S (2014) Phase I clinical trial of pegylated liposomal mitoxantrone plm60-s: pharmacokinetics, toxicity and preliminary efficacy. Cancer Chemo Pharm 74(3):637-646. https://doi.org/10. $1007 / \mathrm{s} 00280-014-2523-8$

24. China NHCo, (2019) Guidelines for the Diagnosis and Treatment of Breast Cancer (Edition 2018). Journal of Multidisciplinary Cancer Management (Electronic Version) 5(3):70-99 ((in Chinese))

25. Sichuan Shenghe Pharmaceutical Limited Company (2009) Instruction of Mitoxantrone Hydrochloride Injection

26. Landys K, Borgstrom S, Andersson T, Noppa H (1985) Mitoxantrone as a first-line treatment of advanced breast cancer. Invest New Drugs 3(2):133-137. https://doi.org/10.1007/BF00174160

27. Neidhart JA, Gochnour D, Roach R, Hoth D, Young D (1986) A comparison of mitoxantrone and doxorubicin in breast cancer. J Clin Oncol 4(5):672-677. https://doi.org/10.1200/JCO.1986.4.5.672

28. Nehoff H, Parayath NN, Domanovitch L, Taurin S, Greish K (2014) Nanomedicine for drug targeting: strategies beyond the enhanced permeability and retention effect. Int J Nanomedicine 9:2539-2555. https://doi.org/10.2147/IJN.S47129

29. Pestalozzi BC, Vass A, Adam H, Horber DH, Schwendener RA, Sauter C (1995) Phase II study of liposome-complexed mitoxantrone in patients with advanced breast cancer. Eur J Cancer 31(6): 1024. https://doi.org/10.1016/0959-8049(95)00010-0

30. Bozzuto G, Molinari A (2015) Liposomes as nanomedical devices. Int J Nanomedicine 10:975-999. https://doi.org/10.2147/ IJN.S68861

31. Li C, Cui J, Li Y, Wang C, Li Y, Zhang L, Zhang L, Guo W, Wang J, Zhang H, Hao Y, Wang Y (2008) Copper ion-mediated liposomal encapsulation of mitoxantrone: The role of anions in drug loading, retention and release. Eur J Pharm Sci 34(4):333-344. https://doi.org/10.1016/j.ejps.2008.05.006

32. ZHANG Lan, DU Yan-ling, LIU Xun-tao, HAO Xiao-fang, WANG Cai-xia, Chun-lei L (2013) Preparation of mitoxantrone hudrocholoride-loaded liposomes and investigation of pharmacodynamics and pharacokinetics. Chin Pharm J 48(17):1475-1479. (In Chinese). https://doi.org/10.11669/cpj.2013.17.014

33. Li C, Cui J, Wang C, Li Y, Zhang H, Wang J, Li Y, Zhang L, Zhang L, Guo W, Wang Y (2008) Encapsulation of mitoxantrone into pegylated SUVs enhances its antineoplastic efficacy. European journal of pharmaceutics and biopharmaceutics : official journal of Arbeitsgemeinschaft fur Pharmazeutische Verfahrenstechnik eV 70(2):657-665. https://doi.org/10.1016/j.ejpb.2008.05.019

34. Banerji U, Kuciejewska A, Ashley S, Walsh G, O’Brien M, Johnston S, Smith I (2007) Factors determining outcome after third line chemotherapy for metastatic breast cancer. Breast 16(4):359-366. https://doi.org/10.1016/j.breast.2007.01.004

35. Porkka K, Blomqvist C, Rissanen P, Elomaa I, Pyrhonen S (1994) Salvage therapies in women who fail to respond to first-line treatment with fluorouracil, epirubicin, and cyclophosphamide for advanced breast cancer. J Clin Oncol 12(8):1639-1647. https:// doi.org/10.1200/JCO.1994.12.8.1639

36. O'Brien MER, Wigler N, Inbar M, Rosso R, Grischke E, Santoro A, Catane R, Kieback DG, Tomczak P, Ackland SP, Orlandi F, Mellars L, Alland L, Tendler C (2004) Reduced cardiotoxicity and comparable efficacy in a phase III trial of pegylated liposomal doxorubicin $\mathrm{HCl}$ (CAELYX ${ }^{\mathrm{TM}} /$ Doxil ${ }^{\circledR}$ ) versus conventional doxorubicin for first-line treatment of metastatic breast cancer. Ann Oncol 15(3):440-449 https://doi.org/10.1093/annonc/mdh097

Publisher's Note Springer Nature remains neutral with regard to jurisdictional claims in published maps and institutional affiliations. 